\title{
Gassensoren auf Basis von Graphen
}

\author{
V. Khomenko, R. Dittrich, Y.Joseph \\ Institute of Electronic and Sensor Materials, Technische Universität Bergakademie Freiberg, Freiberg, Deutschland \\ Kontakt: Yvonne.joseph@esm.tu-freiberg.de
}

\section{Zusammenfassung}

\author{
Schlagwörter: Gassensoren, Graphen, Reduktion von Graphenoxid
}

Chemische Sensoren auf Basis von Graphen wurden durch Beschichtung von Si- Wafern mit Graphenoxid (GO) und dessen Reduktion hergestellt. Lithographisch prozessierte interdigitale Elektroden auf den Si-Substraten mit Elektrodenabständen von $10 \mu \mathrm{m}$ ermöglichten sowohl die sensorischen Messungen als auch eine entsprechende Miniaturisierung. Sowohl chemische als auch thermische Reduktionsprozesse wurden untersucht. Die Reduktion von GO mittels Ascorbinsäure ist eine sehr kostengünstige und umweltfreundliche Herstellungsmethode von Graphenschichten. Der Reduktionsgrad wurde mittels UV/vis-Spektroskopie und Photonenelektronenspektroskopie (XPS) untersucht und die Messungen der Leitfähigkeit bestätigen die graphenähnlichen Eigenschaften der Schichten. Die Untersuchung der gassensorischen Eigenschaften erfolgte in Abhängigkeit vom Reduktionsprozess durch Einsatz von Toluol, 1Propanol, 4-Methyl-2-Pentanon und Wasser als Analyt. Das untersuchte reduzierte GO ist sehr sensibel, Konzentrationen von unter 100 ppm der Analyte konnten detektiert werden.

\section{Motivation}

Graphen ist die Bezeichnung für eine Kohlenstoffmodifikation in zweidimensionaler, bienenwabenartiger Struktur. Diese Kohlenstoffatomlagen weisen in jeder Wabe zwei nicht lokalisierte Doppelbindungen auf. Graphene besitzen außergewöhnliche Eigenschaften, die sie für viele Anwendungen interessant machen. Vor allem die extrem hohe Leitfähigkeit, die hohe spezifische Oberfläche und die extremen mechanischen Eigenschaften führen zur Untersuchung von Graphenen für chemische Sensoren.

Es ist bekannt, dass durch die Adsorption von redoxaktiven Gasmolekülen an den $\pi$-Elektronen der Graphenoberfläche der Schichtwiderstand signifikant erhöht werden kann.

Dadurch ist es möglich, die Schichtleitfähigkeit in Abhängigkeit vom beaufschlagten $\mathrm{Gas} \mathrm{zu}$ verändern. Nichtreaktive organische Analyte wurden nicht untersucht.

\section{Experimente}

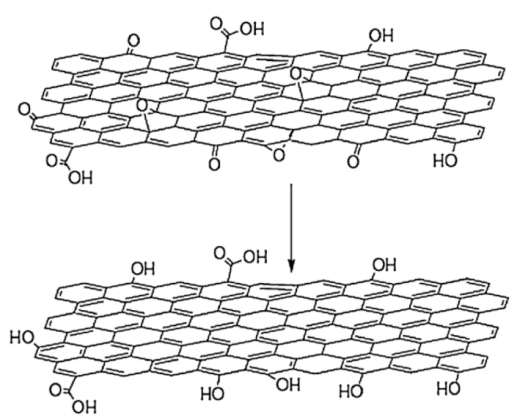

Abb.1: Herstellung von Graphen durch Reduktion von Graphenoxid [1]
Die Graphenschichten wurden durch chemische Reduktion von Graphenoxid mittels Ascorbinsäure und durch thermische Reduktion bei $900^{\circ} \mathrm{C}$ in $\mathrm{Ar} / \mathrm{H}_{2}-$ Atmosphäre hergestellt [2], [3].

Die Beschichtung des Graphenoxides auf den SiSubstraten erfolgte über Spincoating oder drop coating. Sowohl die Menge als auch die Konzentration der Graphenoxidlösung wurden variiert. Abb.1 zeigt schematisch die Reduktion von.

\section{Materialien}

Als Ausgangsmaterial dienten kommerziell verfügbare GO-Lösung (4mg/ml) (Hersteller Graphenea) und Ascorbinsäure der Fa. Merck. Für die chemische Reduktion mit Ascorbinsäure wurde die Konzentration der GOLösung auf $0,1 \mathrm{mg} / \mathrm{ml}$ verringert, wodurch eine bessere Stabilität der reduzierten GO-Lösung erreicht werden konnte. Außerdem waren weitere Bedingungen einzuhalten, um eine Agglomeration von Graphenflocken zu verhindern. Untersuchungen bei Reaktionstemperaturen von $75-95{ }^{\circ} \mathrm{C}$ zeigten, dass optimale Bedingungen für die Reaktion bei $75^{\circ} \mathrm{C}$ vorlagen, ab $80^{\circ} \mathrm{C}$ waren Agglomerationen $\mathrm{zu}$ beobachten. Des Weiteren musste der pH-Wert der Lösung zwischen 9 und 10 konstant gehalten werden, sowohl vor als auch nach der Reduktion, um die reduzierten GO-flocken mit ausreichend $\mathrm{OH}$ Gruppen zu beladen und damit eine elektrostatische Stabilisierung zu erreichen. Nach der Reduktion musste die Lösung mit reduziertem GO schnell auf $5-8^{\circ} \mathrm{C}$ abgekühlt werden. Nur bei Einhaltung dieser drei Bedingungen konnten stabile, nicht agglomerierte, für mehrere Wochen lagerfähige Lösungen hergestellt warden. Als Substratmaterial für die Beschichtung wurden oxidierte, mit Au-Elektroden beschichtete Si-Wafer ver- 
wendet. Die Substrate wurden mit Piranha-Lösung $\left(\mathrm{H}_{2} \mathrm{SO}_{4}: \mathrm{H}_{2} \mathrm{O}_{2}=3: 1\right)$ und SC1-Lösung $\left(\mathrm{NH}_{4} \mathrm{OH}: \mathrm{H}_{2} \mathrm{O}_{2}\right.$ $\left.: \mathrm{H}_{2} \mathrm{O}=1: 1: 5\right)$ gereinigt. Danach wurden die Waferoberfläche mit Aceton $15 \mathrm{~min}$ im Ultraschallbad gereinigt, mit DI-Wasser abgespült und weitere $15 \mathrm{~min}$ in einer Ozonkammer behandelt. Das Ergebnis war eine stark hydrophile Oberfläche, die die Abscheidung der ebenfalls hydrophilen Graphenflocken erleichtert. Versuche, reduziertes GO auf hydrophilen und auch hydrophoben Oberflächen mittels spin coating abzuscheiden, waren nicht erfolgreich. Der Grund könnte sein, dass durch die Reduktion hydrophobe Bereiche und an den Ecken durch noch vorhandene Funktionalitäten partiell hydrophile Bereiche vorlagen, so dass dieses reduzierte GO amphiphilen Charakter hatte.

\section{Chemische Reduktion}

Wie bereits beschrieben wurde die Konzentration der GO-Lösung auf 0,1 mg/ml verdünnt, der $\mathrm{pH}$-Wert auf 910 eingestellt, wofür eine 25\%-ige Ammoniaklösung verwendet wurde, und die Lösung auf $75^{\circ} \mathrm{C}$ erwärmt. Nach Erreichen der Reaktionstemperatur wurde die Ascorbinsäure im Verhältnis 5:1 zur Masse an GO in der Lösung zugegeben. Nach 30 min wurde die Reaktion gestoppt und die Lösung im Eisbad auf $5-8^{\circ} \mathrm{C}$ abgekühlt. Um Reste der Ascorbinsäure aus der Lösung zu entfernen, musste die Lösung filtriert werden, wofür ein Polyamidfilter mit einer Porengröße von $0,2 \mu \mathrm{m}$ verwendet wurde. Die reduzierten GO-Flocken wurden in DI-Wasser redispergiert und erneut filtriert. Der $\mathrm{pH}$ Wert nach der Filtrierung wurde durch Zugabe weniger Mikroliter an Ammoniaklösung weiterhin konsequent auf 9-10 gehalten.

Thermische Reduktion

Um auf oxidierten Si-Wafern feste, dünne Schichten zu erzeugen, kann sowohl spin coating als auch drop coating angewendet werden. Für die thermische Reduktion wurden die mit GO beschichteten Substrate bei $900^{\circ} \mathrm{C}$, 30 min unter Formiergas $\left(\mathrm{Ar} / \mathrm{H}_{2}, 10: 1\right)$ getempert [4]. Dafür wurde der Ofen zuerst auf $900^{\circ} \mathrm{C}$ erhitzt und mit Formiergas gespült. Danach wurden die beschichteten Substrate in die heiße Zone platziert und nach 30 min wieder entfernt und auf Raumtemperatur abgekühlt.

\section{Ergebnisse und Diskussion}

\section{UV/vis- Spektroskopie}

Eine schnelle Methode, um den Reduktionsgrad in einer GO-Lösung zu bestimmen, ist die UV/visSpektroskopie. Der Absorptionspeak von GO zeigt durch die Reduktion eine Rotverschiebung der Wellenlänge von 230nm zu etwa 260nm (Abb.2), wie auch in [5] veröffentlicht. Referenzmessungen an den Ausgangsmaterialien zeigten, dass Ascorbinsäure einen starken Peak in der Nähe des reduzierten GO bei 250nm aufweist. Die für die $\mathrm{pH}$-Stabilisierung verwendete Ammoniaklösung besitzt das Wellenlängenmaximum bei 196nm. Unter Berücksichtigung dieser Ergebnisse ist es ratsam, die Filtrierung nach der chemischen Reduktion mindestens zweimal durchzuführen, um alle Reste Ascorbinsäure zu entfernen und keine Überlagerung der Absorptionspeaks zu erhalten.

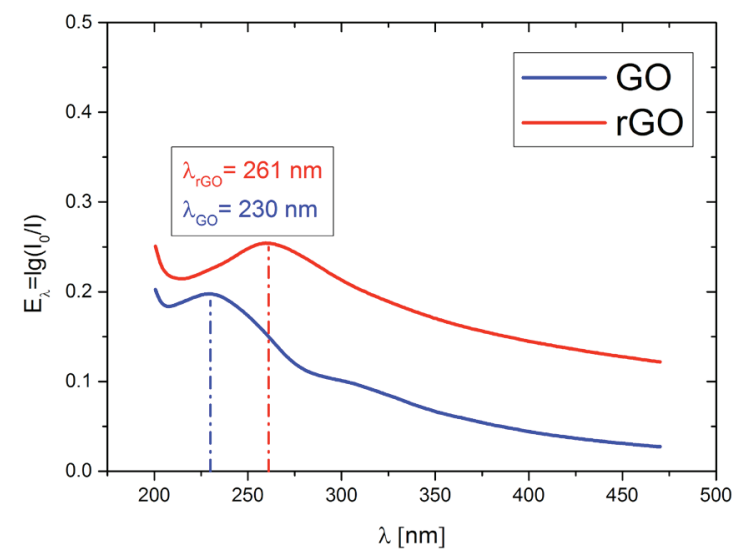

Abb.2: Ergebnisse der UV/vis-Spektroskopie für GO und chemisch reduziertes GO (redispergiert nach dem Waschprozess)

Die beobachtete Rotverschiebung des Absorptionspeaks deutet auf eine Änderung der elektronischen Eigenschaften des GO durch die Reduktion hin. Es ist bekannt dass die Zustandsdichte von Graphen van Hove Singularitäten mit einer Bandlücke von $4,8 \mathrm{eV}$ aufweist $[6,7,8]$. Deshalb besteht eine hohe Wahrscheinlichkeit für Photonen mit Wellenlängen von $260 \mathrm{~nm}(\mathrm{E}=4,7 \mathrm{eV})$ für den elektronischen Bandübergang absorbiert $\mathrm{zu}$ werden, was durch die UV/vis-Untersuchungen bestätigt werden konnte.

\section{XPS-Messungen}

Um den Reduktionsgrad in Zusammenhang mit der chemischen Zusammensetzung und den chemischen Bindungsverhältnissen zu betrachten, wurden XPS_Untersuchungen durchgeführt. Abb.2 zeigt die $\mathrm{C} 1 \mathrm{~s}^{-}$Linie des reinen $\mathrm{GO}$ und des reduzierten GO, 
sowohl nach chemischer als auch nach thermischer Reduktion. Es zeigte sich, dass alle Kohlenstoffatome mit Sauerstoff gebunden waren, also $\mathrm{sp}^{3}$-hybridisiert vorlagen. Die Ergebnisse der quantitativen Analyse sind in Tabelle 1 zusammengefasst. Im vergleich zu reinem GO konnte für beide angewendeten Reduktionsprozesse eine deutliche Desoxidation ermittelt werden.

Abb.3: C1s-Linien von Graphenoxidschichten mit Fits nach chemischer und thermischer Reduktion

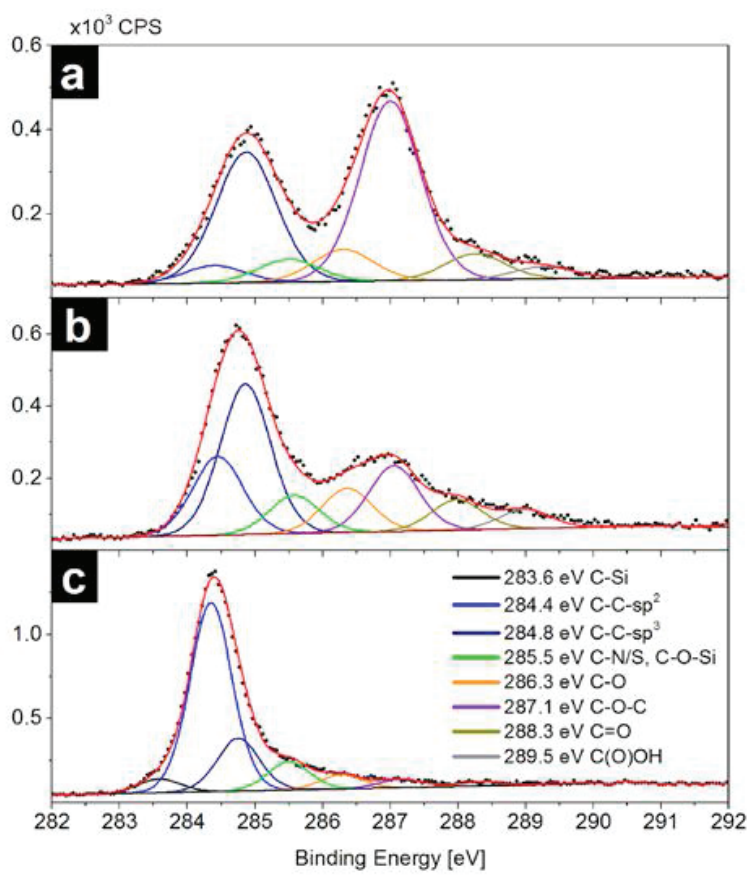

Der Hauptanteil der funktionalen Gruppen sind C-O-CBindungen. Durch die Reduktion wurde der Sauerstoffanteil in der Schicht verringert und die aromatischen Bereiche des Kohlenstoff wiederhergestellt, was zur Erhöhung der elektrischen Leitfähigkeit und der Absorption von sichtbarem Licht (Rotverschiebung des GO nach Reduktion) führte. Es wurde beobachtet, dass es möglich war, durch thermische Reduktion alle Funktionalitäten komplett $\mathrm{zu}$ entfernen. Restgruppen sollten sich nur an den Ecken des GO befinden, da deren Bindungen stabiler sind und mehr Energie notwendig ist, diese zu entfernen. Chemisch reduziertes GO (Abb.3b) zeigte eine Abnahme der C-O-C- Bindungen und ein Anwachsen der $\mathrm{sp}^{2}$-hybridisierten Kohlenstoffatome. Das zeigt, dass die Ascorbinsäure in der Lage war, die $\mathrm{sp}^{3}$-Bindungen aufzubrechen und den Sauerstoff $\mathrm{zu}$ entfernen. Mit Hydrazin als Reduktionsmittel wurde der gleiche Reaktionsmechanismus beobachtet. Außerdem wurde in Abb.3b eine Zunahme der $\mathrm{C}=\mathrm{O}$ und $\mathrm{C}(\mathrm{O}) \mathrm{OH}$ Gruppen beobachtet, was auf Reste an Ascorbinsäure (C6H8O6) nach dem Waschprozess hindeutet.
Tabelle 1: C/O and Csp2 /Csp3 Verhältnisse, ermittelt aus den XPS-Spektren.

\begin{tabular}{|l|l|l|}
\hline & $\mathrm{C} / \mathrm{O}$ & $\mathrm{C}_{\mathrm{sp}}{ }^{2} / \mathrm{C}_{\mathrm{sp}}{ }^{3}$ \\
\hline GO & 2,4 & 0,13 \\
\hline $\begin{array}{l}\text { GO } \\
\text { chem. reduziert }\end{array}$ & 4,8 & 0,6 \\
\hline $\begin{array}{l}\text { GO } \\
\text { therm. reduziert }\end{array}$ & 15,5 & 4,0 \\
\hline
\end{tabular}

Elektrische Messungen

Die elektrischen Leitfähigkeiten der mittels spin coating erzeugten reduzierten GO-Schichten wurden mit der Vierpunkt- Methode ermittelt. Unter Verwendung der beschriebenen Synthese und Charakterisierungsmethoden wurden sensitive Schichten auf Si-Wafern mit interdigitalen Au-Elektroden erzeugt, die reproduzierbare Sensitivitäten gegenüber verschiedenen volatile Verbindungen, wie Toluol, 1-Propanol, 4-Methyl-2-Pentanon oder Wasser aufwiesen (Abb.4).

Für die Messungen wurde trockene, gereinigte Luft durch einen Sättiger mit dem entsprechenden Analyt geleitet und damit die in einer geschlossenen Messzelle kontaktierten Proben beaufschlagt. Um die Reproduzierbarkeit $\mathrm{zu}$ untersuchen wurden jeweils mehrere Zyklen (im Wechsel trockene Luft und Luft mit Analyt) durchgeführt. Die mit diesem Setup gemessenen Änderungen des elektrischen Widerstandes sind in Abb.5 zusammengefasst. Die höchsten Widerstandsänderungen wurden mit Wasser gemessen. Toluol und 4M2P lieferten vergleichbare Ergebnisse und mit 1-Propanol wurden nur geringe Sensoreffekte bestimmt. Die hohe Sensitivität zu Wasser kann durch die (wie in Abb.3) Anteile an Restsauerstoff in den Schichten erklärt werden. Dadurch zeigen die Schichten hydrophilen Charakter und können große Mengen Wassermolelüle adsorbieren. Die adsorbierten Wassermoleküle brechen die Verbindungen zwischen den Schichten und spalten die sp2-hybridisierten Kohlenstoffdomänen, wodurch der elektrische Widerstandes erhöht wird. Ein geringerer, aber signifikanter Effekt wurde mit organischen Analyten gemessen. Da der Reduktionsgrad der hergestellten Graphenschichten unterschiedlich war, varierten auch die Ergebnisse der sensorischen Messungen. Die besten Sensitivitäten wurden mit chemisch reduzierten Schichten erreicht.

Die Diagramme a-d in Abb.4 zeigen die Sensorantworten in Abhängigkeit von der Zeit für die Analyte Wasser, Toluol, 1-Propanol und 4M2P in Konzentrationen von 100-4500ppm. Die Diagramme e-h zeigen die Maxima der Widerstandsänderungen für alle Analyte und Konzentrationen. Mit zunehmender Konzentration läuft die gemessene Widerstandsänderung in eine Sättigung. Die Diffusion in die Mikroporen und zwischen die Graphen- 
schichten dauert länger, wodurch die Sensorantwort langsamer wird.

Tabelle 2: Sensorische Eigenschaften der chemisch reduzierten GO-Schichten

\begin{tabular}{|l|l|l|l|l|}
\hline & Wasser & Toluol & $\begin{array}{l}1- \\
\text { Propa- } \\
\text { nol }\end{array}$ & 4M2P \\
\hline $\begin{array}{l}\text { Reaktionszeit } \\
\text { (min) }\end{array}$ & 6 & 2,3 & 0,7 & 2 \\
\hline Reversibilität & + & - & + & ++ \\
\hline $\begin{array}{l}\text { Sensitivität bis } \\
1500 \text { ppm } \\
(\% / p p m)\end{array}$ & 0,03 & $2 * 10^{-4}$ & $5 \times 10^{-5}$ & $3 \times 10^{-4}$ \\
\hline
\end{tabular}
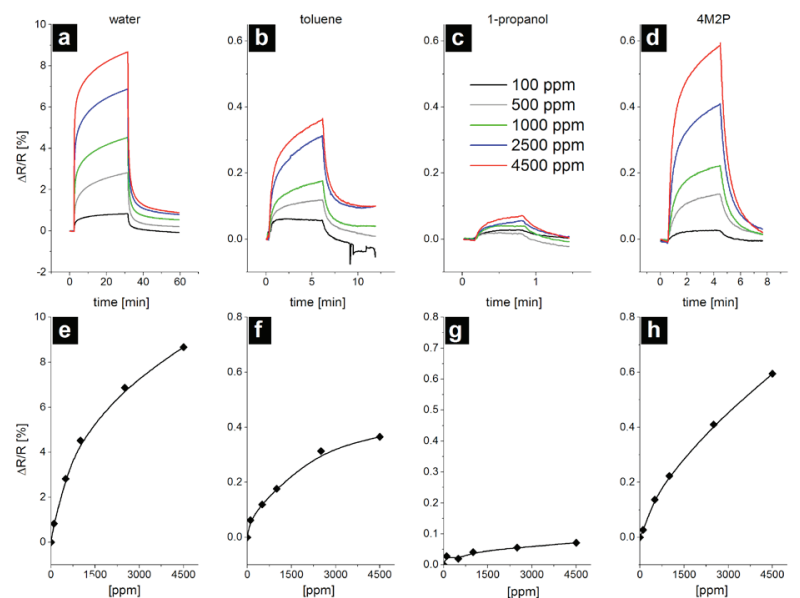

Abb.4: Ergebnisse der sensorischen Messungen von chemisch reduzierten GO-Schichten

Unsere Versuche führten $\mathrm{zu}$ partiell reduzierten GOSchichten mit $\mathrm{sp}^{2}$-Kohlenstoffdomänen, die eine elektrische Leitfähigkeit ermöglichten. Durch die Adsorption von Molekülen auf den Oberflächen, zwischen den Schichten und auch an den Ecken der reduzierten GOSchichten wurde der Widerstand der Schichten erhöht und damit eine geringere Leitfähigkeit ermittelt.

\section{Literatur}

[1] Basu, S.; Bhattacharyya, P. Recent developments on graphene and graphene oxide based solid state gas sensors. In Sensors and Actuators B(2012): Chemical $173,1-21$.
[2] J. T. Robinson, F. K. Perkins, E. S. Snow, Z. Wei und P. E. Sheehan, Nano Lett., p. 3137-3140, 2008.

[3] M. J. Fernández-Merino, L. Guardia, J. I. Paredes, S. Villar-Rodil, P. Solís-Fernández, A. Martínez-Alonso und J. M. D. Tascón, J. Phys. Chem. C, p. 6426-6432, 2010.

[4] X. Wang, L. Zhi und K. Müllen, Nano Lett., p. 323-327, 2008.

[5] D. Li, M. B. Muller, S. Gilje, R. B. Kaner, G. G. Wallace,

Nat Nano 3 (2) (2008) 101-105.

[6] K. S. Novoselov, S. V. Morozov, Mohinddin, T. M. G., L. A. Ponomarenko, D. C. Elias, R. Yang, I. I. Barbolina, P. Blake, T. J. Booth, D. Jiang, J. Giesbers, E. W. Hill, A. K. Geim, physica status solidi (b) 244 (11) (2007) 4106-4111.

[7] K. A. Mkhoyan, A. W. Contryman, J. Silcox, D. A. Stewart, G. Eda, C. Mattevi, S. Miller, M. Chhowalla, Nano Letters 9 (3) (2009) 1058-1063.

[8] D. W. Boukhvalov, Physical Chemistry Chemical Physics 12 (47) (2010) 15367 